\title{
An $\omega$-categorical structure with amenable automorphism group
}

\author{
A. Ivanov *
}

\begin{abstract}
We analyse $\omega$-categorical precompact expansions of particular $\omega$-categorical structures from the viewpoint of amenability of their automorphism groups.

2010 Mathematics Subject Classification: 03C15, 03E15

Keywords: Amenable groups, Countably categorical structures.
\end{abstract}

\section{Introduction}

A group $G$ is called amenable if every $G$-flow (i.e. a compact Hausdorff space along with a continuous G-action) supports an invariant Borel probability measure. If every $G$-flow has a fixed point then we say that $G$ is extremely amenable. Let $M$ be a relational countably categorical structure which is a Fraïssé limit of a Fraïssé class $\mathcal{K}$. In particular $\mathcal{K}$ coincides with $\operatorname{Age}(M)$, the class of all finite substructures of $M$. By Theorem 4.8 of [9] the group $A u t(M)$ is extremely amenable if and only if the class $\mathcal{K}$ has the Ramsey property and consists of rigid elements. Here the class $\mathcal{K}$ is said to have the Ramsey property if for any $k$ and a pair $A<B$ from $\mathcal{K}$ there exists $C \in \mathcal{K}$ so that each $k$-coloring

$$
\xi:\left(\begin{array}{l}
C \\
A
\end{array}\right) \rightarrow k
$$

*The author is supported by Polish National Science Centre grant DEC2011/01/B/ST1/01406 
is monochromatic on some $\left(\begin{array}{l}B^{\prime} \\ A^{\prime}\end{array}\right)$ from $C$ which is a copy of $\left(\begin{array}{l}B \\ A\end{array}\right)$, i.e.

$$
C \rightarrow(B)_{k}^{A}
$$

We remind the reader that $\left(\begin{array}{l}C \\ A\end{array}\right)$ denotes the set of all substructures of $C$ isomorphic to $A$. This result has become a basic tool to amenability of automorphism groups. To see whether $A u t(M)$ is amenable one usually looks for an expansion $M^{*}$ of $M$ so that $M^{*}$ is a Fraïssé structure with extremely amenable $A u t\left(M^{*}\right)$. Moreover it is usually assumed that $M^{*}$ is a precompact expansion of $M$, i.e. every member of $\mathcal{K}$ has finitely many expansions in $\operatorname{Age}\left(M^{*}\right)$, see [9], [10], [12], [1] and [13]. Theorem 9.2 from [1] and Theorem 2.1 from [13] describe amenability of $A u t(M)$ in this situation. The question if there is a countably categorical structure $M$ with amenable automorphism group which does not have expansions as above was formulated by several people. We mention very similar Problems 27, 28 in [2] where precompactness is replaced by $\omega$ categoricity and finite homogenity.

We think that in order to construct a required example one can use the ideas applied in [7] where we construct an $\omega$-categorical structure so that its theory is not G-compact and it does not have AZ-enumerations. These ideas develop ones applied in slightly different forms in [8] and [6] for some other questions. Moreover Casanovas, Pelaez and Ziegler suggest in [3] a general method which simplifies and generalises our approach from [6], [7] and [8]. The basic object of this construction is a particular theory $T_{E}$ of equivalence relations $E_{n}$ on $n$-tuples. The paper 3 . pays attention to several model-theoretic properties of $T_{E}$.

Below we study $T_{E}$ from the viewpoint of (extreme) amenability of its expansions. Then we apply our results to a construction of a family of concrete candidates for an example of an $\omega$-categorical structure with amenable automorphism group and without $\omega$-categorical precompact expansions with extremely amenable automorphism groups. We will in particular show that these structures have the following unusual combination of properties:

- the automorphis group is amenable;

- it does not satisfy Hrushovski's extension property;

- it does not have an order expansion with the Ramsey property.

In fact we will show a slightly stronger version of the latter property.

\section{Equivalence relations}

We start with a very interesting reduct of the structure from [7]. This is $T_{E}$ mentioned in the introduction. It has already deserved some attention in model-theoretic community, see [3].

Let $L_{0}=\left\{E_{n}: 0<n<\omega\right\}$ be a first-order language, where each $E_{n}$ is a relational symbol of arity $2 n$. Let $\mathcal{K}_{0}$ be the class of all finite $L_{0}$-structures $C$ where each relation 
$E_{n}(\bar{x}, \bar{y})$ determines an equivalence relation on the set (denoted by $\left(\begin{array}{l}C \\ n\end{array}\right)$ ) of unordered $n$-element subsets of $C$. In particular for every $n$ the class $\mathcal{K}_{0}$ satisfies the sentence

$$
\forall \bar{x} \bar{y}\left(E_{n}\left(x_{1}, \ldots, x_{n}, y_{1}, \ldots, y_{n}\right) \rightarrow \bigwedge\left\{E_{n}\left(y_{1}, \ldots, y_{n}, x_{\sigma(1)}, \ldots, x_{\sigma(n)}\right): \sigma \in \operatorname{Sym}(n)\right\}\right)
$$

Note that for $C \in \mathcal{K}_{0}, E_{n}$ is not satisfied by $\bar{a}, \bar{b}$ if one of these tuples has a repetition. Thus for $n>|C|$ we put that no $2 n$-tuple from $C$ satisfies $E_{n}(\bar{x}, \bar{y})$. It is easy to see that $\mathcal{K}_{0}$ is closed under taking substructures and the number of isomorphism types of $\mathcal{K}_{0}$-structures of any size is finite.

Let us verify the amalgamation property for $\mathcal{K}_{0}$. Given $A, B_{1}, B_{2} \in \mathcal{K}_{0}$ with $B_{1} \cap B_{2}=A$, define $C \in \mathcal{K}_{0}$ as $B_{1} \cup B_{2}$ with the finest equivalence relations among those which obey the following rules. When $n \leq\left|B_{1} \cup B_{2}\right|$ and $\bar{a} \in\left(\begin{array}{c}B_{1} \\ n\end{array}\right) \cup\left(\begin{array}{c}B_{2} \\ n\end{array}\right)$ we put that the $E_{n}$-class of $\bar{a}$ in $C$ is contained in $\left(\begin{array}{c}B_{1} \\ n\end{array}\right) \cup\left(\begin{array}{c}B_{2} \\ n\end{array}\right)$. We also assume that all $n$-tuples meeting both $B_{1} \backslash B_{2}$ and $B_{2} \backslash B_{1}$ are pairwise equivalent with respect to $E_{n}$. In particular if $n \geq \max \left(\left|B_{1}\right|,\left|B_{2}\right|\right)$ we put that all $n$-element $n$-tuples from $C$ are pairwise $E_{n}$-equivalent.

It is easy to see that this amalgamation also works for the joint embedding property.

Let $M_{0}$ be the countable universal homogeneous structure for $\mathcal{K}_{0}$. It is clear that in $M_{0}$ each $E_{n}$ defines infinitely many classes and each $E_{n}$-class is infinite. Let $T_{E}=T h\left(M_{0}\right)$.

Theorem 1.2 which we prove below, shows that $M_{0}$ cannot be treated by the methods of [9]. It states that the group $\operatorname{Aut}\left(M_{0}\right)$ is amenable but the structure $M_{0}$ does not have a linear ordering so that the corresponding age has the order property and the Ramsey property.

It is worth noting that this statement already holds for the $\left\{E_{1}, E_{2}\right\}$-reduct of $M_{0}$, see the proof below. Thus our theorem also gives some interesting finitely homogeneous examples. On the other hand amenability of $\operatorname{Aut}\left(M_{0}\right)$ is a harder task than the corresponding statement in the reduct's case.

The statement that $\operatorname{Aut}\left(M_{0}\right)$ is amenable is a consequence of a stronger property, namely Hrushovski's extension property for partial isomorphisms. This is defined for Fraïssé limits as follows.

Definition 1.1 A universal ultrahomogeneous structure U satisfies Hrushovski's extension property if for any finite family of finite partial isomorphisms between substructures of $U$ there is a finite substructure $F<U$ containing these substructures so that any isomorphism from the family extends to an automorphism of $F$.

Proposition 6.4 of [11 states that the structure $U$ has Hrushovski's extension property if and only if $A u t(U)$ has a dense subgroup which is the union of a countable chain of compact subgroups. The latter implies amenability by Theorem 449C of [4].

Theorem 1.2 (a) The structure $M_{0}$ satisfies Hrushovski's extension property. In particular the group Aut $\left(M_{0}\right)$ is amenable. 
(b) The structure $M_{0}$ does not have any expansion by a linear order so that $T h\left(M_{0},<\right)$ admits elimination of quantifiers and the age of $\left(M_{0},<\right)$ satisfies the Ramsey property.

The proof uses some material from [5]. We now describe it.

Let $L$ be a finite relational language. We say that an $L$-structure $F$ is irreflexive if for any $R \in L$, any tuple from $F$ satisfying $R$ consists of pairwise distinct elements. An irreflexive $L$-structure $F$ is called a link structure if $F$ is a singleton or $F$ can be enumerated $\left\{a_{1}, \ldots, a_{n}\right\}$ so that $\left(a_{1}, \ldots, a_{n}\right)$ satisfies a relation from $L$.

Let $\mathcal{S}$ be a finite set of link structures. Then an $L$-structure $N$ is of link type $\mathcal{S}$ if any substructure of $N$ which is a link structure is isomorphic to a structure from $\mathcal{S}$.

An $L$-structure $F$ is packed if any pair from $F$ belongs to a link structure which is a substructure of $F$.

If $\mathcal{R}$ is a finite family of packed irreflexive $L$-structures, then an $L$-structure $F$ is called $\mathcal{R}$-free if there does not exist a weak homomorphism (a map preserving the predicates) from a structure from $\mathcal{R}$ to $F$.

Proposition 4 and Theorem 5 of [5] state that for any family of irreflexive link structures $\mathcal{S}$ and any finite family of irreflexive packed $L$-structures $\mathcal{R}$ the class of all irreflexive finite $L$-structures of link type $\mathcal{S}$ which are $\mathcal{R}$-free, has the free amalgamation property and Hrushovski's extension property for partial isomorphisms.

We will use a slightly stronger version of this statement concerning permorphisms. A partial mapping $\rho$ on $U$ is called a $\chi$-permorphism, if $\chi$ is a permutation of symbols in $L$ preserving the arity and for every $R \in L$ and $\bar{a} \in \operatorname{Dom}(\rho)$ we have

$$
\bar{a} \in R \Leftrightarrow \rho(\bar{a}) \in R^{\chi}
$$

The following statement is a version of Lemma 6 from [5].

Lemma 1.3 Let $L$ be a finite language, $\chi_{1}, \ldots, \chi_{n}$ be arity preserving permutations of $L$ and $\mathcal{S}$ be a finite $\left\{\chi_{i}\right\}_{i \leq n}$-invariant family of irreflexive link structures. Let $\mathcal{R}$ be a finite family of finite irreflexive packed L-structures of link type $\mathcal{S}$ so that $\mathcal{R}$ is invariant under all $\chi_{i}$. Let $A$ be a finite structure which belongs to the class, say $K$, of $L$-structures of link type $\mathcal{S}$ which are $\mathcal{R}$-free. Let $\rho_{i}, i \leq n$, be parial $\chi_{i}$-permorphisms of $A$.

Then there is a finite $B \in K$ containing $A$ so that each $\rho_{i}$ extends to a permutation of $B$ which is a $\chi_{i}$-permorphism.

Proof of Theorem 1.2. (a) For each $n>0$ enumerate all $E_{n}$-classes. Consider the expansion of $M_{0}$ by distinguishing each $E_{n}$-class by a predicate $P_{n, i}$ according the enumeration. Let $L^{*}$ be the language of all predicates $P_{n, i}$ and let $M^{*}$ be the $L^{*}$-structure defined on $M_{0}$. For every finite sublanguage $L^{\prime} \subseteq L^{*}$ let $M^{*}\left(L^{\prime}\right)$ be the $L^{\prime}$-reduct of $M^{*}$ defined by these interpretations.

We denote by $\mathcal{K}\left(L^{\prime}\right)$ the class of all finite $L^{\prime}$-structures with the properties that for any arity $l$ represented by $L^{\prime}$ : 
- any $l$-relation is irreflexive and invariant with respect to all permutations of variables,

- any two relations of $L^{\prime}$ of arity $l$ have empty intersection.

Let $\mathcal{S}\left(L^{\prime}\right)$ be the set of all link structures of $\mathcal{K}\left(L^{\prime}\right)$ satisfying these two properties. Thus $\mathcal{K}\left(L^{\prime}\right)$ is of link type $\mathcal{S}\left(L^{\prime}\right)$.

Claim 1. For every finite sublanguage $L^{\prime} \subseteq L^{*}$ the structure $M^{*}\left(L^{\prime}\right)$ is a universal structure with respect to the class $\mathcal{K}\left(L^{\prime}\right)$.

It is easy to see that any structure from $\mathcal{K}\left(L^{\prime}\right)$ can be expanded to a structure from $\mathcal{K}_{0}$ so that $L^{\prime}$-predicates become classes of appropriate $E_{n}$ 's.

Claim 2. For every finite sublanguage $L^{\prime} \subseteq L^{*}$ the structure $M^{*}\left(L^{\prime}\right)$ is an ultrahomogeneous structure.

Let $f$ be an isomorphism between finite substructures of $M^{*}\left(L^{\prime}\right)$. We may assume that $\operatorname{Dom}(f)$ contains tuples representing all $M^{*}\left(L^{\prime}\right)$-predicates of $L^{\prime}$ (some disjoint tuples can be added to $\operatorname{Dom}(f)$ in a suitable way). Then $f$ extends to an automorphism of $M_{0}$ fixing the classes of appropriate $E_{n}$ 's which appear in $L^{\prime}$. Thus this automorphism is an automorphism of $M^{*}\left(L^{\prime}\right)$ too.

Claim 3. For each finite sublanguage $L^{\prime} \subseteq L^{*}$ let $\mathcal{R}\left(L^{\prime}\right)$ be the family of all packed $L^{\prime}$-structures of the form $\left(\left\{a_{1}, \ldots, a_{n}\right\}, P_{n, i}, P_{n, j}\right)$, where $i \neq j, P_{n, i}=\left\{\left(a_{1}, \ldots, a_{n}\right)\right\}$ and $P_{n, j}=\left\{\left(a_{\sigma(1)}, \ldots, a_{\sigma(n)}\right)\right\}$ for some permutation $\sigma$. Then the class $\mathcal{K}\left(L^{\prime}\right)$ is the class of all irreflexive finite $L^{\prime}$-structures of link type $\mathcal{S}\left(L^{\prime}\right)$, which are $\mathcal{R}\left(L^{\prime}\right)$-free.

The claim is obvious. By Proposition 4 and Theorem 5 of [5] we now see that $\mathcal{K}\left(L^{\prime}\right)$ is closed under substructures, has the joint embedding property, the free amalgamation property, Hrushovski's extension property and its version for permorphisms, i.e. the statement of Lemma 1.3.

By Claim 1 and Claim 2 the structure $M^{*}\left(L^{\prime}\right)$ is the universal homogeneous structure of $\mathcal{K}\left(L^{\prime}\right)$. In particular any tuple of finite partial isomorphisms (permorphisms) of $M^{*}\left(L^{\prime}\right)$ can be extended to a tuple of automorphisms (permorphisms) of a finite substructure of $M^{*}\left(L^{\prime}\right)$.

Note that the same statement holds for the structure $M^{*}$. To see this take any tuple $f_{1}, \ldots, f_{k}$ of finite partial isomorphisms (resp. $\chi_{i}$-permorphisms) of $M^{*}$ (assuming that $\chi_{i}$ are finitary). Let $r$ be the size of the union $\bigcup_{i<k} \operatorname{Dom}\left(f_{i}\right)$ and $L^{\prime}$ be the minimal (resp. $\left\{\chi_{i}\right\}_{i \leq k}$-invariant) sublanguage of $L^{*}$ of arity $r$ containing of all relations of $M^{*}$ which meet any tuple from $\bigcup_{i<k} \operatorname{Dom}\left(f_{i}\right)$. Then there is a finite substructure $A$ of $M^{*}\left(L^{\prime}\right)$ containing $\bigcup_{i \leq k} \operatorname{Dom}\left(f_{i}\right)$ so that each $f_{i}$ extends to an automorphism (resp. $\chi_{i}$-permorphism) of $\bar{A}$.

Let $r^{\prime}$ be the size of $A$. Let $L^{\prime \prime}$ be a sublanguage of $L^{*}$ so that $L^{\prime} \subseteq L^{\prime \prime}$ and for each arity $l \leq r^{\prime}$ the sublanguage $L^{\prime \prime} \backslash L^{\prime}$ contains exactly one $l$-relation, say $P_{l, n_{l}}$ (fixed by $\left.\left\{\chi_{i}\right\}_{i \leq k}\right)$. Since $M^{*}$ is the universal homogeneous structure of $\mathcal{K}\left(L^{\prime \prime}\right)$ the substructure $A$ can be chosen so that any $l$-subset of $A$ which does not satisfy any relation from $L^{\prime}$, does satisfy $P_{l, n_{l}}$.

As a result any automorphism (permorphism) of $A$ preserves the relations of $L^{\prime \prime \prime} \backslash L^{\prime}$ for any $L^{\prime \prime \prime} \subset L^{*}$ containing $L^{\prime \prime}$. Thus it extends to an automorphism (permorphism) of $M^{*}\left(L^{\prime \prime \prime}\right)$. In paricular it extends to an automorphism (permorphism) of $M^{*}$. 
As in Proposition 6.4 of [11] we see that $A u t\left(M^{*}\right)$ has a dense subgroup which is the union of a countable chain of compact subgroups. In particlar we arrive at the following statement.

Claim 4. $\operatorname{Aut}\left(M^{*}\right)$ is amenable.

Since each automorphism of $M_{0}$ is a permorphism of $M^{*}$ and vice versa, we also see that $\operatorname{Aut}\left(M_{0}\right)$ has a dense subgroup which is the union of a countable chain of compact subgroups. In particular $\operatorname{Aut}\left(M_{0}\right)$ is amenable.

(b) Consider a linearly ordered expansion $\left(M_{0},<\right)$ together with the corresponding age, say $\mathcal{K}^{<}$. Assume that $\mathcal{K}^{<}$has the Ramsey property.

Note that $\mathcal{K}^{<}$does not contain any three-element structure of the form $a<b<c$, where $a$ and $c$ belong to the same $E_{1}$-class which is distinct from the $E_{1}$-class of $b$. Indeed, otherwise repeating the argument of Theorem 6.4 from [9], we see that in any larger structure from $\mathcal{K}^{<}$we can colour two-elements structures $a<b$ with $\neg E_{1}(a, b)$, so that there is no monochromatic three-element structure of the form above.

As a result we see that any $E_{1}$-class of $\left(M_{0},<\right)$ is convex. We now claim that the following structure $B$ can be embedded into $\left(M_{0},<\right)$.

Let $B=\left\{a_{1}<a_{2}<a_{3}<a_{4}<b_{1}<b_{2}\right\}$, where the $E_{1}$-classes of all elements are pairwise distinct, but the pairs $\left\{a_{1}, a_{2}\right\}$ and $\left\{b_{1}, b_{2}\right\}$ are $E_{2}$-equivalent. We assume that in all other cases any two distinct pairs from $B$ belong to distinct $E_{2}$-classes. Moreover we assume that for each $k=3,4,5$ all $k$-subsets from $B$ belong to the same $E_{k}$-class. In particular the ordered structures defined on $\left\{a_{1}, a_{2}, a_{3}, a_{4}\right\}$ and $\left\{a_{3}, a_{4}, b_{1}, b_{2}\right\}$ are isomorphic. Let $A$ represent this isomorphism class.

Since $M_{0}$ is the universal homogeneous structure with respect to $\mathcal{K}_{0}$, taking any tuple $a_{1}^{\prime}<a_{2}^{\prime}<a_{3}^{\prime}<a_{4}^{\prime}<b_{1}^{\prime}<b_{2}^{\prime}$ with pairwise distinct $E_{1}$-classes we can find $B$ in $M_{0}$ as a half of a copy of a structure from $\mathcal{K}_{0}$ consisting of 12 elements where each $E_{1}$-class is represented by a pair $\left(a_{i}^{\prime}, a_{i}\right)$ or $\left(b_{i}^{\prime}, b_{i}\right)$.

To show that the Ramsey property does not hold for the age of $\left(M_{0},<\right)$ take any finite substructure $C$ of this age which extends $B$. Fix any enumeration of $E_{2}$-classes ocurring in $C$. Then colour a copy of $A$ red if the class of the first two elements is enumerated before the class of the last pair. Otherwise colour such a copy green. It is clear that $C$ does not contain a structure isomorphic to $B$ so that all substructures of type $A$ are of the same colour.

Remark 1.4 It is worth noting that the class $\mathcal{K}_{0}^{<}$of all linearly ordered members of $\mathcal{K}_{0}$ has JEP and AP, i.e. there is a generic expansion of $M_{0}$ by a linear ordering. To see AP we just apply the amalgamation described above together with the standard amalgamation of orderings.

\section{$2 \quad$ Adding dense linear orders}

In order to obtain a structure with the properties as in Section 1, but without Hrushovski's extension property we use a general approach from [3]. In fact our

starting point is Corollary 2.8 from [3] that sets $\left(\begin{array}{c}M_{0} \\ n\end{array}\right) / E_{n}$ (definable in $T h^{e q}\left(M_{0}\right)$ ) are 
stably embedded in $M_{0}$.

We remind the reader that a 0 -definable predicate $P$ of a theory $T$ is called stably embedded if every definable relation on $P$ is definable with parameters from $P$. If $M$ is a saturated model of $T$ then $P$ is stably embedded if and only if every elementary permutation of $P(M)$ extends to an automorphism of $M$ (see remarks after Definition 2.4 in [3]). We now formulate Lemma 3.1 from [3].

Let $T$ be a complete theory with two sorts $S_{0}$ and $S_{1}$. Let $\tilde{T}_{1}$ be a complete expansion of $T \uparrow S_{1}$. Assume that $S_{1}$ is stably embedded. Then

(1) $\tilde{T}=T \cup \tilde{T}_{1}$ is a complete theory;

(2) $S_{1}$ is stably embedded in $\tilde{T}$ and $\tilde{T} \uparrow S_{1}=\tilde{T}_{1}$.

(3) if $T$ and $\tilde{T}_{1}$ are $\omega$-categorical, then $\tilde{T}$ is also $\omega$-categorical.

We now describe our variations of $M_{0}$. Let us fix $S_{n}=\left(\begin{array}{c}M_{0} \\ n\end{array}\right) / E_{n}, n \in \omega$, and consider them as a sequence of stably embedded sorts in $T h^{e q}\left(M_{0}\right)$ (this is Corollary 2.8 of [3]). We can distinguish relations $\left\{a_{1}, . ., a_{n}\right\} \in e$, where $e \in S_{n}$ is an $E_{n}$-class, $n \in \omega$.

We also fix a subset $P \subset \omega \backslash\{1,2\}$ and consider the language

$$
L_{P}^{S}=\left\{E_{n}: 0<n \in \omega\right\} \cup\left\{S_{n},<_{S_{n}}: n \in P\right\},
$$

where $<_{S_{n}}$ are binary relations on $S_{n}$. Let $\tilde{T}_{1}$ be the theory of sorts $\left\{S_{n}: n \in \omega\right\}$, where for every $n \in P$ the relation $<_{S_{n}}$ is a dense linear order without ends. When $n \notin P$ the sort $S_{n}$ is considered as a pure set. This is an $\omega$-categorical theory for each $S_{n}$. Applying Lemma 3.1 from [3] we define the complete theory $T_{P}^{S}=T_{E} \cup \tilde{T}_{1}$ which is $\omega$-categorical and every sort $S_{n}$ is stably embedded into $T_{P}^{S}$.

We now define an one-sorted version of $T_{P}^{S}$. Its countable model will be the example anounced in Introduction.

Let $L_{P}=\left\{E_{n}: 0<n \in \omega\right\} \cup\left\{<_{n}: n \in P\right\}$ be a first-order language, where each $E_{n}$ and $<_{n}$ is a relational symbol of arity $2 n$. The $L_{P}$-structure $M$ is built by the Fraïssé's construction. Let us specify a class $\mathcal{K}_{P}$ of finite $L_{P}$-structures, which will become the class of all finite substructures of $M$.

Assume that in each $C \in \mathcal{K}_{P}$ each relation $E_{n}(\bar{x}, \bar{y})$ determines an equivalence relation on the set (denoted by $\left(\begin{array}{l}C \\ n\end{array}\right)$ ) of unordered $n$-element subsets of $C$. As before for $C \in \mathcal{K}_{P}$ and $n>|C|$ we put that no $2 n$-tuple from $C$ satisfies $E_{n}(\bar{x}, \bar{y})$.

For $n \in P$ the relations $<_{n}$ are irreflexive and respect $E_{n}$,

$$
\forall \bar{x}, \bar{y}, \bar{u}, \bar{w}\left(E_{n}(\bar{x}, \bar{y}) \wedge E_{n}(\bar{u}, \bar{w}) \wedge<_{n}(\bar{x}, \bar{u}) \rightarrow<_{n}(\bar{y}, \bar{w})\right) .
$$

Every $<_{n}$ is interpreted by a linear order on the set of $E_{n}$-classes. Therefore we take the corresponding axioms (assuming below that tuples consist of pairwise distinct elements):

$$
\begin{gathered}
\forall \bar{x}, \bar{y}\left(<_{n}(\bar{x}, \bar{y}) \rightarrow \neg E_{n}(\bar{x}, \bar{y})\right) ; \\
\forall \bar{x}, \bar{y}, \bar{z}\left(<_{n}(\bar{x}, \bar{y}) \wedge<_{n}(\bar{y}, \bar{z}) \rightarrow<_{n}(\bar{x}, \bar{z})\right) ; \\
\forall \bar{x}, \bar{y}\left(\neg E_{n}(\bar{x}, \bar{y}) \rightarrow<_{n}(\bar{x}, \bar{y}) \vee<_{n}(\bar{y}, \bar{x})\right) .
\end{gathered}
$$


Lemma 2.1 (1) The class $\mathcal{K}_{P}$ satisfies the joint embedding property and the amalgamation property.

(2) Let $M$ be the generic structure of $\mathcal{K}_{P}$. For every $n>0$ let $M_{n}=\left(\begin{array}{c}M \\ n\end{array}\right) / E_{n}$.

Then Th(M) is w-categorical, admits elimination of quantifiers, and $<_{n}$ is a dense linear ordering on $M_{n}$ without ends (when $n \in P$ ). The structure $M$ is an expansion of $M_{0}$.

(3) Let $\rho_{i}, i \leq k$, be a sequence of finitary maps on $M_{i}$ which respect $<_{i}$ for $i \in P$. Then there is an automorphism $\alpha \in A u t(M)$ realising each $\rho_{i}$ on its domain.

Proof. (1) Given $A, B_{1}, B_{2} \in \mathcal{C}$ with $B_{1} \cap B_{2}=A$, define $C \in \mathcal{K}$ as $B_{1} \cup B_{2}$. The relations $E_{n},<_{n}, n \leq\left|B_{1} \cup B_{2}\right|$, are defined so that $C \in \mathcal{K}, B_{1}<C, B_{2}<C$ and the following conditions hold. Let $n \leq\left|B_{1} \cup B_{2}\right|$. We put that all $n$-element $n$-tuples meeting both $B_{1} \backslash B_{2}$ and $B_{2} \backslash B_{1}$ are pairwise equivalent with respect to $E_{n}$. We additionally demand that they are equivalent to some tuple from some $B_{i}, i \in\{1,2\}$, if $n \leq \max \left(\left|B_{1}\right|,\left|B_{2}\right|\right)$. If for some $i \in\{1,2\},\left|\left(\begin{array}{c}B_{i} \\ n\end{array}\right) / E_{n}\right|=1$, then we put that all $n$-tuples $\bar{c} \in B_{1} \cup B_{2}$ meeting $B_{i}$ are pairwise $E_{n}$-equivalent. We additionally arrange that they are equivalent to some tuple from $B_{3-i}$ if $n \leq\left|B_{3-i}\right|$. If $n \geq \max \left(\left|B_{1}\right|,\left|B_{2}\right|\right)$ then all $n$-element $n$-tuples from $C$ are pairwise $E_{n}$-equivalent. We take $E_{n}$ to be the minimal equivalence relation satisfying the conditions above. In particular if $n$-tuples $\bar{b}_{1}$ and $\bar{b}_{2}$ are $E_{n}$-equivalent to the same $n$-tuple from $A$, then $E_{n}\left(\bar{b}_{1}, \bar{b}_{2}\right)$.

We can now define the linear orderings $<_{n}$ on $C / E_{n}$ for $n \in P$. There is nothing to do if $\left|\left(\begin{array}{l}C \\ n\end{array}\right) / E_{n}\right|=1$. In the case when for some $i=1,2,\left|\left(\begin{array}{c}B_{i} \\ n\end{array}\right) / E_{n}\right|=1$, the relation $<_{n}$ is defined by its restriction to $B_{3-i}$. When $\left|\left(\begin{array}{c}B_{1} \\ n\end{array}\right) / E_{n}\right| \neq 1 \neq\left|\left(\begin{array}{c}B_{2} \\ n\end{array}\right) / E_{n}\right|$ and $V_{1}, V_{2}$ is a pair of two $<_{n}$-neighbours among $E_{n}$-classes having representatives both in $\left(\begin{array}{c}B_{1} \\ n\end{array}\right)$ and $\left(\begin{array}{c}B_{2} \\ n\end{array}\right)$, we amalgamate the $<_{n}$-linear orderings between $V_{1}$ and $V_{2}$ assuming that all elements of $\left(\begin{array}{c}B_{1} \\ n\end{array}\right) / E_{n} \cap\left[V_{1}, V_{2}\right]$ are less than those from $\left(\begin{array}{c}B_{2} \\ n\end{array}\right) / E_{n} \cap\left[V_{1}, V_{2}\right]$.

We appropriately modify this procedure for intervals open from one side. It is clear that this defines $<_{n}$-ordering on $\left(\begin{array}{l}C \\ n\end{array}\right) / E_{n}$.

(2) The statement that $T h(M)$ admits elimination of quantifiers and is $\omega$-categorical, follows from (1). This also implies that $M$ is a natural expansion of $M_{0}$.

To see the second statement of this part of the lemma it is enough to show that for $n \in P$ and any two sequences $V_{1}<_{n} \ldots<_{n} V_{k}$ and $V_{1}^{\prime}<_{n} \ldots<_{n} V_{k}^{\prime}$ from $M_{n}$ there is an automorphism of $M$ taking each $V_{i}$ to $V_{i}^{\prime}$. To see this we use the fact that $M$ is the Fraïssé limit of $\mathcal{K}_{P}$. This allows us to find pairwise disjoint representatives of classes $V_{1}, \ldots, V_{k}$, say $\bar{a}_{1}, \ldots, \bar{a}_{k}$, and classes $V_{1}^{\prime}, \ldots, V_{k}^{\prime}$, say $\bar{a}_{1}^{\prime}, \ldots, \bar{a}_{k}^{\prime}$, so that for every $m \neq n$ all $m$-tuples of the substructures $\bar{a}_{1} \cup \ldots \cup \bar{a}_{k}$ and $\bar{a}_{1}^{\prime} \cup \ldots \cup \bar{a}_{k}^{\prime}$ are $E_{m}$-equivalent. Moreover all $n$-tuples meeting at least two $\bar{a}_{s}, \bar{a}_{t}$ or $\bar{a}_{s}^{\prime}, \bar{a}_{t}^{\prime}$ also belong to a single $E_{n^{-}}$ class. Taking an appropriate isomorphism induced by these representatives we extend it to a required automorphism.

(3) We develop the argument of (2). For each $\rho_{i}$ find a sequence $\bar{a}_{1}, \ldots, \bar{a}_{t}$ of pairwise disjoint tuples from $M$ representing the $E_{i}$-classes of the domain and of the range of $\rho_{i}$. We may assume that for any $j \neq i$ all $j$-tuples of the union $\Omega_{i}=\bar{a}_{1} \cup \ldots \cup \bar{a}_{t}$ belong to the same $E_{j}$-class. Moreover all $i$-tuples meeting at least two $\bar{a}_{l}, \bar{a}_{m}$ also form a single $E_{i}$-class. Thus $\rho_{i}$ can be realised by a partial map on $\Omega_{i}$. We may 
arrange that all $\Omega_{i}$ are pairwise disjont and do not have common $E_{n}$-classes. Thus all $\rho_{i}$ can be realised by a partial isomorphism on the union of these $\Omega_{i}$. Since $M$ is ultrahomogeneous, this partial isomorphism can be extended to an automorphism of $M$.

Let us consider $M$ in the language $L_{P}^{S}$, i.e.

$$
\left(M, E_{1}, \ldots, E_{n}, \ldots\right) \cup\left(M_{1}, *_{1}\right) \cup \ldots \cup\left(M_{n}, *_{n}\right) \cup \ldots,
$$

where $*_{n}=<_{n}$ for $n \in P$ and disappears for $n \notin P$. By Lemma 2.1(3) the structure of all sorts $\left\{M_{n}: n \in \omega\right\}$ coincides with the theory $\tilde{T}_{1}$ of sorts $\left\{S_{n}: n \in \omega\right\}$ of the theory $T_{P}^{S}$. This implies the following corollary.

Corollary 2.2 The theory of $M$ in the language $L_{P}^{S}$ coincides with $T_{P}^{S}$. In particular the sets $M_{n}$ are stably embedded into $M$.

We see that for $n \in P$ any automorphism of $\left(M_{n},<_{n}\right)$ can be realized by an automorphism of $M$. Assume that $2 n \notin P$. Let us consider automorphisms $\alpha$ of $M_{n}$ which are increasing, i.e. for any $V \in M_{n}, V<_{n} \alpha(V)$.

Take an orbit of $\alpha$ of the following form:

$$
\ldots \rightarrow \bar{a}_{-1} \rightarrow \bar{a}_{0} \rightarrow \bar{a}_{1} \rightarrow \bar{a}_{2} \rightarrow \bar{a}_{3} \rightarrow \bar{a}_{4} \rightarrow \ldots
$$

and consider $E_{2 n}$-classes of tuples $\bar{a}_{i} \bar{a}_{i+1}$. Applying ultrahomogenity and the choice of $n$ it is easy to see that $\alpha$ can be taken so that there are four $E_{2 n}$-classes, say $V_{1}, V_{2}, V_{3}, V_{4}$, represented by consecutive pairs of tuples $\bar{a}_{1}, \bar{a}_{2}, \bar{a}_{3}, \bar{a}_{4}, \bar{a}_{5}, \bar{a}_{6}$ and $\alpha$ acts on them by $\mathbb{Z} / 4 \mathbb{Z}$ :

$$
\text { if } \bar{a}_{1} \bar{a}_{2} \in V_{1} \text {, then } \bar{a}_{2} \bar{a}_{3} \in V_{2}, \bar{a}_{3} \bar{a}_{4} \in V_{3} \text { and } \bar{a}_{4} \bar{a}_{5} \in V_{4} \text {, }
$$

where $\bar{a}_{1} \bar{a}_{2}$ and $\bar{a}_{5} \bar{a}_{6}$ are $E_{2 n}$-equivalent.

Slightly generalising this situation we will say that a sequence $\bar{a}_{1}, \bar{a}_{2}, \bar{a}_{3}, \bar{a}_{4}, \bar{a}_{5}, \bar{a}_{6}$ is $<_{n}$-increasing of type $\mathbb{Z} / 4 \mathbb{Z}$ if the following conditions are satisfied:

- tuples $\bar{a}_{1} \bar{a}_{2}, \bar{a}_{2} \bar{a}_{3}$ and $\bar{a}_{3} \bar{a}_{4}$ are of the same isomorphism type,

- tuples $\bar{a}_{1} \bar{a}_{2} \bar{a}_{3} \bar{a}_{4}$ and $\bar{a}_{3} \bar{a}_{4} \bar{a}_{5} \bar{a}_{6}$ are of the same isomorphism type and

- tupes $\bar{a}_{1} \bar{a}_{2}$ and $\bar{a}_{5} \bar{a}_{6}$ are $E_{2 n}$-equivalent but not $E_{2 n}$-equivalent to $\bar{a}_{3} \bar{a}_{4}$.

Let $L^{\prime}$ be an extension of $L_{P}$ and $M^{\prime}=(M, \overline{\mathbf{r}})$ be an $L^{\prime}$-expansion of $M$ with quantifier elimination. We do not demand that $\overline{\mathbf{r}}$ is finite, we only assume that $M^{\prime}$ is a precompact expansion. It is clear that $M^{\prime}$ induces a subgroup of $\operatorname{Aut}\left(M_{n},<_{n}\right)$.

We will say that a sequence $\bar{a}_{1}, \bar{a}_{2}, \bar{a}_{3}, \bar{a}_{4}, \bar{a}_{5}, \bar{a}_{6}$ is $<_{n}$-increasing of type $\mathbb{Z} / 4 \mathbb{Z}$ in $M^{\prime}$ if the definition above holds under the assumption that the isomorphism types appeared in the definition are considered with respect to the relations of $M^{\prime}$. 
Theorem 2.3 Let $M$ be the generic structure of $\mathcal{K}_{P}$ where $P \neq \emptyset$. Then the group $G=\operatorname{Aut}(M)$ is amenable, $M$ does not satisfy Hrushovski's extension property and does not have an extremely amenable ultrahomogeneous expansion by a linear ordering.

Let $M^{\prime}$ be a precompact expansion of $M$ with quantifier elimination. If Aut $\left(M^{\prime}\right)$ is extremely amenable, then for any $n \in P$ with $2 n \notin P$ the structure $M^{\prime}$ does not have an $<_{n}$-increasing sequence of type $\mathbb{Z} / 4 \mathbb{Z}$.

The main point of this theorem is that although in different arities the structures induced by $M$ are completely independent, any expansion $M^{\prime}$ as in the formulation simultaneously destroys $M$ in all arities $n \in P$ with $2 n \notin P$.

The proof below uses the proof of Theorem 1.2.

Proof of Theorem 2.3. For each $n>1$ enumerate all $E_{n}$-classes. Consider the expansion of $M$ by distinguishing each $E_{n}$-class by a predicate $P_{n, i}$ according the enumeration. Let $L^{*}$ be the language of all predicates $P_{n, i}$ and let $M^{*}$ be the $L^{*}$ structure defined on $M$. By Claims 1 - 4 of the proof of Theorem 1.2 the structure $M^{*}$ has Hrushovski's extension property and $A u t\left(M^{*}\right)$ is amenable.

Let us consider the structure $\left(M_{n},<_{n}\right)$, where $n \in P$. As it is isomorphic to $(\mathbb{Q},<)$, the group $\operatorname{Aut}\left(M_{n},<_{n}\right)$ is extremely amenable $([9])$.

Since each automorphism of $M$ preserves all $<_{i}, i \in P$, it is easy to see that there is a natural homomorphism from $A u t(M)$ to the product

$$
\prod_{i \in P} \operatorname{Aut}\left(M_{i},<_{i}\right) \times \prod_{i \notin P} \operatorname{Sym}\left(M_{i}\right)
$$

and $\operatorname{Aut}\left(M^{*}\right)$ is the kernel of it. By Corollary 2.2 this homomorphism is surjective. Now by Theorem 449C of [4] we have the following claim.

The group Aut $(M)$ is amenable.

To see that $M$ does not satisfy Hrushovski's extension property take $n \in P$ and let us consider any triple of pairwise disjoint $n$-tuples $\bar{a}, \bar{b}, \bar{c}$ representing pairwise distinct elements of $M_{n}$ so that

$$
\bar{a}<_{n} \bar{b}<_{n} \bar{c} .
$$

Then the map $\phi$ fixing $\bar{a}$ and taking $\bar{b}$ to $\bar{c}$ cannot be extended to an automorphism of a finite substructure of $M$.

Consider a linearly ordered expansion $(M,<)$ with quantifier elimination. To see that $\operatorname{Aut}(M,<)$ is not extremely amenable just apply the argument of statement (b) of Theorem 1.2. Since at arity 2 the structure $M$ coincides with $M_{0}$ it works without any change.

To prove the second part of the theorem we slightly modify that argument.

Let $n \in P$ and $2 n \notin P$. Let a structure $B$ consist of $6 n$ elements forming a sequence

$$
\bar{a}_{1}<_{n} \bar{a}_{2}<_{n} \bar{a}_{3}<_{n} \bar{a}_{4}<_{n} \bar{b}_{1}<_{n} \bar{b}_{2},
$$

where the tuples $\bar{a}_{1} \bar{a}_{2}$ and $\bar{b}_{1} \bar{b}_{2}$ are $E_{2 n}$-equivalent but not of the same $E_{2 n}$-class with $\bar{a}_{3} \bar{a}_{4}$. We assume that the tuples $\bar{a}_{1} \bar{a}_{2}, \bar{a}_{2} \bar{a}_{3}$, and $\bar{a}_{3} \bar{a}_{4}$ are of the same isomorphism class in $M^{\prime}$ and the substructure $\bar{a}_{1} \bar{a}_{2} \bar{a}_{3} \bar{a}_{4}<M^{\prime}$ is isomorphic to $\bar{a}_{3} \bar{a}_{4} \bar{b}_{1} \bar{b}_{2}<M^{\prime}$. Since 
$\operatorname{Aut}\left(M^{\prime}\right)$ is extremely amenable, these structures are rigid and the corresponding isomorphisms are uniquely defined on these tuples.

Let $A$ represent the isomorphism class of $\bar{a}_{1} \bar{a}_{2} \bar{a}_{3} \bar{a}_{4}$ in $M^{\prime}$. Let us show that the Ramsey property does not hold for the age of $M^{\prime}$. Take any finite substructure $C$ of this age which extends $B$. Fix any enumeration of $E_{2 n}$-classes ocurring in $C$. Then colour a copy of $A$ red if the class of the first two $n$-tuples is enumerated before the class of the last pair. Otherwise colour such a copy green. It is clear that $C$ does not contain a structure isomorphic to $B$ so that all substructures of type $A$ are of the same colour.

\section{References}

[1] O.Angel, A.Kechris and R.Lyone, Random orderings and unique ergodicity of automorphism groups, to appear in Jour. Europ. Math. Soc., ArXiv: 1208.2389

[2] M.Bodirsky, M.Pinsker and T.Tsankov, Decidability of definability, In: Proceedings of the 26-th Annual IEEE Symposium on Logic in Computer Science (LICS'11), IEEE Computer Society, 2011, 321 - 328.

[3] E.Casanovas, R.Pelaez and M.Ziegler, On many-sorted w-categorical theories, Fund Math. 214(2011), 285 - 294.

[4] D.H.Fremlin, Measure Theory, vol 4. Topological measure spaces. Part I,II. Torres Fremlin, Colchester, 2006

[5] B. Herwig, Extending partial isomorphisms for the small index property of many $\omega$-categorical structures, Israel J. Math. 107 (1998), 93 - 123.

[6] A.Ivanov, Automorphisms of homogeneous structures, Notre Dame Journal of Formal Logic, 46 (2005), no.4, $419-424$.

[7] A.Ivanov, A countably categorical theory which is not G-compact, Siberian Adv. in Math. 20(2010), 75 - 82.

[8] A.Ivanov and H.D.Macpherson, Strongly determined types, Ann. Pure and Appl. Logic 99(1999), 197 - 230.

[9] A.Kechris, V.Pestov and S.Todorcevic, Fraïssé limites, Ramsey theory, and topological dynamics of automorphism groups, GAFA, 15(2005), 106 -189.

[10] A.Kechris and M.Sokić, Dynamical properties of the automorphism groups of the random poset and random distributive lattice, Fund Math. 218(2012), 69 - 94.

[11] A.Kechris and Ch.Rosendal, Turbulence, amalgamation, and generic automorphisms of homogeneous structures, Proc. London Math. Soc. (3) 94(2007), 302 350. 
[12] L.N. van Thé, More on Kechris-Pestov-Todorcevic correspondence: precompact expnsions, Fund. Math. 222(2013), 19 - 47.

[13] A.Zucker, Amenability and unique ergodicity of automorphism groups of Frä̈ssé structures, arXiv:1304.2839.

Institute of Mathematics, University of Wrocław, pl.Grunwaldzki 2/4, 50-384 Wrocław, Poland,

E-mail: ivanov@math.uni.wroc.pl 